\title{
Spektrum Autoimmun - wissenswert, kompakt, anregend
}

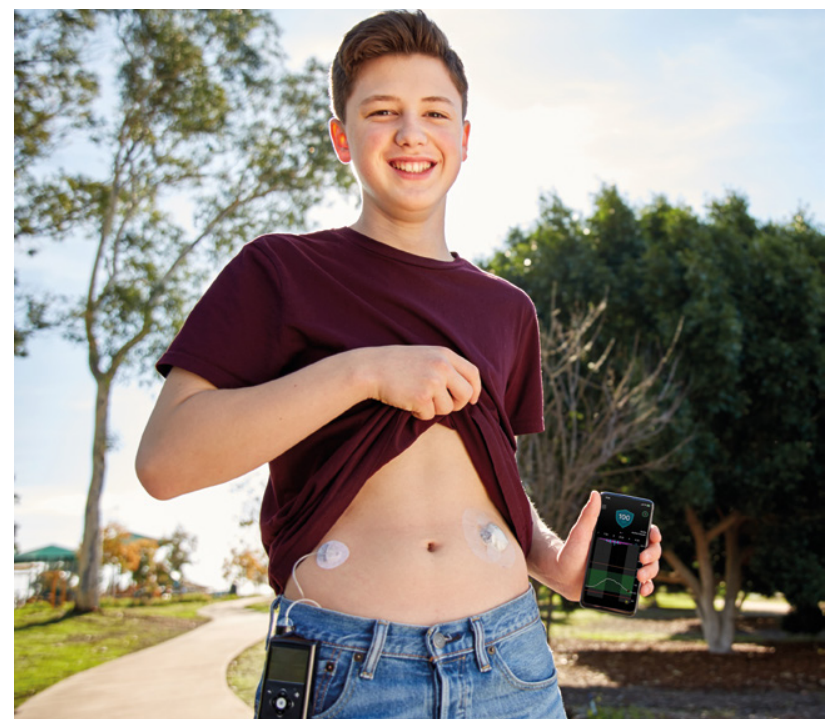

Früher Insulinpumpen-Einsatz bei Kindern mit Typ-1-Diabetes bringt viele Vorteile. (c) Medtronic $\mathrm{GmbH}$

\section{Deutsche Diabetes Gesellschaft FokUs}

\section{Frühzeitiger Einsatz einer Insulinpumpe verbessert Behandlung von Kindern mit Diabetes Typ 1}

Bei Kindern und Jugendliche, die rasch nach der Diagnose ihres Typ-1-Diabetes mit einer Insulinpumpe therapiert werden, kommt es seltener zu lebensbedrohlichen Stoffwechselentgleisungen und weniger Krankhausaufenthalten als bei einem verzögerten Pumpeneinsatz. Eine MulticenterStudie, an der Mitglieder der Arbeitsgemeinschaft für Pädiatrische Diabetologie (AGPD) und der Deutschen Diabetes Gesellschaft (DDG) beteiligt waren, belegt den Vorteil des frühzeitigen Starts einer Insulinpumpentherapie.

Die aktuelle Studie verglich dafür die Ergebnisse zwischen einem frühen Einsatz der Insulinpumpentherapie innerhalb der ersten sechs Monate nach der Erstdiagnose mit einem verzögerten Pumpenbeginn im zweiten oder dritten Jahr nach Diagnosestellung. Insgesamt wurden die Daten von 8332 Patienten aus 311 Diabeteszentren in Deutschland, Österreich, Schweiz und Luxemburg analysiert. «Die Patienten waren zwischen 6 Monate und 15 Jahre alt, als die Diagnose gestellt wurde», erläutert Studienautor Professor Dr. Reinhard Holl, Kinder-Endokrinologe und Diabetologe an der Universität Ulm. Alle Patienten wurden mindestens ein Jahr lang mit einer Insulinpumpe therapiert.

Positive Effekte auf Blutzucker-, Blutdruckund Cholesterinwerte

Die Daten belegen einen signifikanter Unterschied bei den Blutzuckerwerten: Der durchschnittliche HbA1c-Wert betrug in der frühen Pumpengruppe $7,9 \%$ gegenüber $8,0 \%$ in der späten Pumpengruppe - und lag damit günstiger. Kinder, die frühzeitig eine Insulinpumpe bekommen hatten, erlitten zudem seltener ein gefährliches Koma aufgrund starker Unterzuckerung, sie mussten insgesamt weniger häufig mit Komplikationen im Krankenhaus behandelt werden. Darüber hinaus konnten bei einer frühzeiti- gen Insulinpumpentherapie positive Effekte auf Blutdruck- und Cholesterinwerte festgestellt werden. Der Body-Mass-Index war bei beiden Gruppen annähernd gleich, der frühe Einsatz einer Insulinpumpe ist nicht mit Gewichtszunahme verbunden. «Insgesamt liefern unsere Ergebnisse klare Hinweise, dass ein früher Insulinpumpen-Einsatz bei Kindern mit Typ-1-Diabetes zu besseren Behandlungsergebnissen führt», resümiert PD Dr. Thomas Kapellen. Weitere Hintergründe müsse man näher erforschen, fügt der Vorsitzende der AGPD hinzu.

\section{Literatur}

Kamrath C, et al.: Lancet Child Adolesc Health. 2021;5(1): $17-25$.

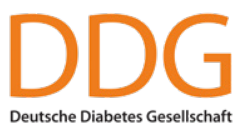

www.deutsche-diabetes-gesellschaft.de 


\section{Studie will Entstehung von Typ-1-Diabetes bei Kindern verhindern}

In frühen Stadien von Typ-1-Diabetes treten sogenannte Inselautoantikörper im Blut auf. Sie sind ein Anzeichen dafür, dass das Immunsystem die insulinproduzierenden Zellen angreift. Aus früheren Studien wissen die Forschenden, dass die Darmflora bei Kindern, die diese Antikörper entwickeln, gestört sein kann. Die Sint1a-Studie zielt darauf ab, das Auftreten von Inselautoantikörpern bei Kindern mit einem erhöhten genetischen Risiko für Typ-1-Diabetes zu verhindern. Dazu wird innen das Probiotikum Bifidobacterium Infantis (B. infantis EVC001) zusammen mit der täglichen Nahrung verabreicht. Dies soll eine gesunde und ausgewogene Entwicklung ihrer Darmflora unterstützen und das Immunsystem positiv beeinflussen.

Sint1a baut auf eine andere Studie namens Point auf. In der Point-Studie erhalten Kinder Insulinpulver, das ebenfalls gemeinsam mit der Nahrung verabreicht wird. «Wir gehen davon aus, dass das Immunsystem der Mund- und Darmschleimhaut für die Prävention von Krankheiten wie Typ-1-Diabetes

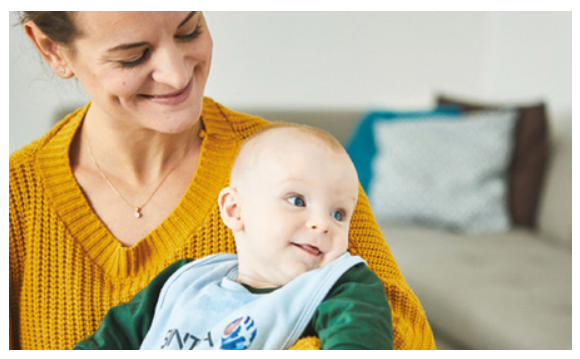

Um an der SINT1A-Studie teilzunehmen, dürfen Kinder nicht älter als 6 Wochen sein. (A)Andreas Pfohl, NOZY Films GmbH

eine wichtige Rolle spielt», erklärt Prof. Bonifacio, Studienleiter in Dresden. «Die PointStudie nutzt den Darm, um das Immunsystem mit Insulin vertraut zu machen und eine Autoimmunreaktion dagegen zu verhindern. Sintla basiert auf dem Wissen, dass eine gesunde Darmflora Entzündungen reduziert und dies dem Immunsystem hilft, gefährliche Antigene von ungefährlichen zu unterscheiden.» Auf diese Weise will die Sint1a-Studie die Wahrscheinlichkeit verringern, dass Kinder mit einem hohen genetischen Risiko für Typ-1-Diabetes, Immunreaktionen auslösen, die zu einer Autoimmunität führen. «Sollten die Ergebnisse beider Studien erfolgreich sein, werden wir sie für eine optimierte synergistische Typ-1-Diabetes-Präventionsstrategie miteinander kombinieren. Typ-1-Diabetes wäre dann kein unausweichliches Schicksal mehr, sondern eine Krankheit, die wir mit den richtigen Maßnahmen verhindern können.»

Die Teilnahme an den Sint1a- und Point-Studien erfordert den Nachweis eines erhöhten genetischen Typ-1-Diabetes-Risikos Wenn mindestens ein Elternteil oder ein Geschwisterkind bereits an Typ-1-Diabetes erkrankt ist, kann ein Baby bis zum Alter von 7 Tagen deutschlandweit an der Typ-1-Diabetesrisiko-Früherkennung des GPPAD-Netzwerks teilnehmen.

Weitere Informationen zum Typ1-DiabetesRisiko-Screening unter https://www.gppad.org/ de/standorte/.

TECHNISCHE UNIVERSITÄT DRESDEN

www.tu-dresden.de

Kassenärztliche Bundesvereinigung

\section{Ambulante spezialfachärztliche Versorgung: Anpassungen beschlossen}

Der Gemeinsame Bundesausschuss hat die jährlichen Anpassungen für die ambulante spezialfachärztliche Versorgung (ASV) sowie weitere Detailänderungen beschlossen. Akzeptiert das Bundesgesundheitsministerium den Beschluss, tritt er Ende Mai/ Anfang Juni in Kraft.

Eine wesentliche Detailänderung betrifft die Anlage 1.1b Rheumatologische Erkrankungen: Im ASV-Team wurden die Fachgruppen Frauenheilkunde und Urologie gestrichen, die bis-

lang zur Behandlung Erwachsener hinzugezogen werden konnten (Ebene 3). Beim ASVRheumateam zur Behandlung von Kindern und Jugendlichen wurde analog die Fachgruppe Frauenheilkunde gestrichen; die Fachgruppe Urologie gehörte hier nicht zum ASVTeam. Für bereits bestehende ASV-Teams bedeutet das, dass beide Fachgruppen nur noch bis zum zu erwarteten Inkrafttreten des G-BABeschlusses zum ASV-Team gehören und in die Behandlung einbezogen werden dürfen.

Interdisziplinär in Praxen und Kliniken

Die ASV ist ein Versorgungsbereich für Patienten, die an einer seltenen oder schweren Erkrankung mit besonderem Krankheitsverlauf leiden. Interdisziplinäre Teams aus Praxis- und Klinikärzten übernehmen die ambulante hochspezialisierte Behandlung. Wie der Versorgungsbereich funktioniert, regelt die ASV-Richtlinie des Gemeinsamen Bundesausschusses. In den Anlagen werden die allgemeinen Regeln für jede ASVIndikation konkretisiert.
Zudem wurde für die Behandlung rheumatologischer Erkrankungen in der ASV der Patientenkreis erweitert: Mit Aufnahme des ICD-10-Kodes M11. können nun auch Patientinnen und Patienten mit bestimmten Kristall-Arthropathien in der ASV behandelt werden, die aufgrund eines schweren Verlaufs auf eine hohe Expertise angewiesen sind. Eher milde Verläufe werden weiterhin in der Regelversorgung behandelt.

Des Weiteren kann das ASV-Rheumateam künftig auch bestimmte Patientinnen und Patienten mit Sarkoidose behandeln. Die entsprechenden ICD-10-Kodes wurden ebenfalls der Anlage 1.1b Rheumatologische Erkrankungen zugeordnet. Die Anlage 2e Sarkoidose bleibt bestehen. 


\section{Exzellenzcluster Precision Medicine in Chronic Inflammation}

\section{Durchbruch in der Entwicklung neuer anti-entzündlicher Therapien}

Chronisch-entzündliche Darmerkrankungen (CED) wie Morbus Crohn und Colitis ulcerosa führen zu dauerhaft wiederkehrendem Durchfall, Fieber und Schmerzen sowie schwerwiegenden psychischen Belastungen. In Deutschland sind rund 320000 Menschen von CED betroffen. Bei ihnen führt eine gestörte Immunantwort zu einer Entzündung im Magen-Darm-Trakt, die in Schüben immer wieder aufflammt. Es gibt nur wenige zugelassene Medikamente für diese Erkrankungen und diese wirken bei weitem nicht bei allen Betroffenen, sondern jeweils nur bei einigen. Es besteht daher ein großer Bedarf an Therapien, die über neue Wirkmechanismen die chronischen Darmentzündungen bekämpfen. Forschende des Exzellenzclusters Precision Medicine in Chronic Inflammation (PMI) haben vor einiger Zeit ein Fusionsprotein konstruiert, das zum jetzt untersuchten Wirkstoffkandida-

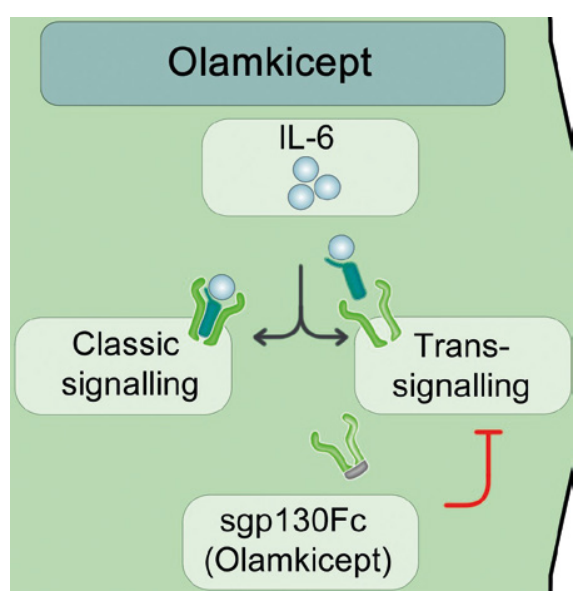

Olamkicept fängt den Komplex aus IL-6 und löslichem Rezeptor aus dem Blut ein und blockiert dadurch den Trans-Signalweg ohne die positiven Wirkungen von IL-6 über den «klassischen» Weg zu stören.

(c) Gastroenterology, AGA Institute ten «Olamkicept» weiterentwickelt wurde. Olamkicept wirkt über einen speziellen Signalweg eines wichtigen Botenstoffs des Immunsystems, des sogenannten Interleukin (IL)-6. Dieses Signalmolekül wird bei einer Entzündung vom Körper vermehrt ausgeschüttet und hilft bei der Regulation der ablaufenden Immunprozesse. Wenn jedoch ein Medikament alle Wirkungen von IL-6 blockiert, dämpft dies zwar sehr erfolgreich die Entzündung, aber gleichzeitig kann es zu schweren Nebenwirkungen kommen. Durch die Blockade von IL-6 kann das Immunsystem so stark unterdrückt werden, dass der Körper deutlich anfälliger gegenüber Infektionen wird. Dies ist bei bereits erhältlichen Antikörpern gegen IL-6 oder seinen spezifischen Rezeptor der Fall.

«Das Besondere an Olamkicept ist, dass es gezielt nur einen Teil der IL-6-Wirkung über den sogenannten IL-6-Trans-Signalweg hemmt. Wir gehen davon aus, dass der Trans-Signalweg besonders bei chronischen Entzündungen aktiv ist», erklärt Professor Philip Rosenstiel, Vorstandsmitglied im Exzellenzcluster PMI und Direktor des Instituts für klinische Molekularbiologie.

Beim «klassischen» Signalweg bindet IL-6 spezifisch an seinen Rezeptor, der nur auf der Oberfläche bestimmter Zellen vorkommt. Gemeinsam binden sie dann an ein weiteres Oberflächenprotein auf derselben Zelle, das sogenannte gp130-Protein, und lösen so eine Reaktion in der Zelle aus. Aber der IL6-Rezeptor kommt auch in löslicher Form im Blut vor. Beim alternativen IL-6-Trans-Signalweg bindet IL- 6 an diesen im Blut zirkulierenden Rezeptor und dann an gp130, das auf allen Zellen vorkommt. So kann IL-6 theore- tisch auf jede Zelle im Körper wirken. Ein Fusionsprotein, der Prototyp von Olamkicept, fängt den Komplex aus IL-6 und löslichem Rezeptor aus dem Blut ein und blockiert dadurch den Trans-Signalweg, ohne die positiven Wirkungen von IL-6 über den «klassischen» Weg zu stören.

Zur Verbesserung seiner Wirkung und zur großtechnischen Herstellung wurde das Protein von der Kieler Biotechnologiefirma CONARIS Research Institute AG zum Medikamentenkandidaten Olamkicept weiterentwickelt.

In zahlreichen Tiermodellen haben sgp130Fc und Olamkicept bereits ihre Wirksamkeit bewiesen und bekämpften erfolgreich chronische Entzündungen, ohne das Immunsystem zu unterdrücken. Die schweren Nebenwirkungen einer Therapie, die IL-6 blockiert, blieben aus. Nun hat das Kieler Forschungsteam das Medikament erstmals an einer kleinen Gruppe von Erkrankten mit Colitis Ulcerosa oder Morbus Crohn getestet. Im menschlichen Körper zeigte der Wirkstoff die gewünschte Wirkung : Der IL-6-Trans-Signalweg wird blockiert, die Entzündung dadurch gedämpft. Wie die Wirksamkeit und das Nebenwirkungsprofil in einer größeren Patientenkohorte aussieht, wird derzeit in verblindeten und Placebo-kontrollierten klinischen Studien der Phase II untersucht.

\section{Literatur}

Schreiber S, et al:. Gastroenterology. 2021;S0016-5085 (21)00467-4.

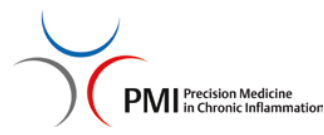

www.precisionmedicine.de

Arbeitsgemeinschaft der Wissenschaftlichen Medizinischen Fachgesellschaften e.V.

\section{Leitlinie Colitis ulcerosa aktualisiert}

Zu Jahresbeginn wurde die überarbeitete Leitlinie zu Colitis ulcerosa publiziert. Diese Leitlinie wird gemäß des sogenannten «living guidelines»-Konzeptes regelmäßig aktualisiert. Das bedeutet, dass die Überarbeitung in kürzeren Abständen als üblicherweise erfolgt, um eine hohe Aktualität der Empfehlungen zu gewähr- leisten In der aktuellen Fassung wurden unter anderem Empfehlungen bezüglich des monoklonalen Antikörpers Ustekinumab aufgenommen, der kürzlich für die Behandlung einer Colitis ulcerosa mit mittel- bis hochgradiger Entzündungsaktivität, bei der eine konventionellen Therapie nicht anschlägt, zugelassen wurde.
Colitis ulcerosa - Living Guideline, Registernummer 021 - 009, Anhang Zusatzmaterial, eingestellt am 26.01.2021

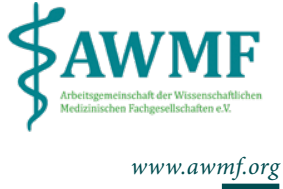




\section{Salzkonsum reguliert Autoimmunerkrankung}

Die Auswirkungen von übermäßigem Salzkonsum bei Autoimmun- und Entzündungskrankheiten wie Multipler Sklerose (MS) wurden in der Vergangenheit an Tiermodellen mit «Experimental Autoimmune Encephalomyelitis> (EAE), einer MS-ähnlichen Erkrankung, untersucht. Der übermäßige Salzkonsum verschlimmerte demnach die Erkrankung. Jetzt konnte Gurumoorthy Krishnamoorthy, Leiter der Forschungsgruppe «Neuroinflammation und Mukosale Immunologie» am Max-Planck-Institut für Biochemie, mit seinem Team gegensätzliche Ergebnisse gewinnen.

Der Forschungsgruppenleiter erklärt: «Für unsere Studien haben wir ein anderes Mausmodell verwendet, das spontan MSähnliche Symptome entwickelt. Wir haben keinen Hinweis darauf, dass der erhöhte Salzkonsum bei den Tieren die Erkrankung begünstigt oder verschlimmert.» Die Wissenschaftlerinnen und Wissenschaftler konnten sogar zeigen, dass der erhöhte Salzkonsum die Entwicklung der Autoimmunerkrankung unterdrückt.

Für die Analyse fokussierten sich die Forschenden auf die Blut-Hirn-Schranke. Sie ist eine wichtige Barriere zwischen dem Blutkreislauf und dem zentralem Nervensystem. Sie verhindert, dass Stoffe, aber auch Immunzellen, aus dem Blut unkontrolliert in das zentrale Nervensystem übertreten. Bei dieser Diffusionsbarriere helfen sogenannte Tight Junctions. Das sind Membranmoleküle, die zwischen Zellen eine enge Verbindung herstellen. Bei den Tieren, die vermehrt Salz konsumierten, waren die Serumspiegel des Glucocorticoid-Hormons Kortikosteron erhöht. Dieser erhöhte Kortikosteron-Spiegel hat zu einer erhöhten Expression der Tight-Junction-Moleküle in den Endothelzellen geführt. Dadurch wurde die Blut-Hirn-Schranke gestärkt und der Eintritt von entzündlichen TZellen in das Nervensystem wurde blockiert.
«Unsere Ergebnisse zeigen, dass ein moderat erhöhter Salzkonsum vielfältige und potentiell vorteilhafte Effekte auf die Autoimmunität des zentralen Nervensystems bei Mäusen hat», berichtet Krishnamoorthy. Er gehe davon aus, dass der entgegengesetzte Effekt zu den früheren Studien mit den verschiedenen Tiermodellen zusammen hängt, bei denen die Blut-Hirn-Schranke durch Injektion von Pertussis-Toxin künstlich geöffnet wurde. Das sei bei dem aktuell untersuchten Krankheitsmodell nicht der Fall und komme dem frühen Stadium einer MS-Erkrankung beim Menschen näher.

\section{Literatur}

Na SY, et al.: Proc Natl Acad Sci U S A. 2021;118(12): e2025944118.

max-planck-institut für biochemie

www.biochem.mpg.de

\section{Deutsche Gesellschaft für Rheumatologie}

\section{Strukturiertes Behandlungsprogramm für Rheumapatienten kommt}

Gute Aussichten für Patienten mit rheumatoider Arthritis: Der Gemeinsame Bundesausschuss (G-BA) hat jetzt für die chronischen Entzündung der Gelenke ein strukturiertes Behandlungsprogramm (DMP) beschlossen. Es soll durch koordinierte Betreuung und Schulung der Patienten dazu beitragen, die Therapieziele zu erreichen: möglichst weitgehende Entzündungs-und Beschwerdefreiheit oder zumindest geringe Krankheitsaktivität.

Für die Entwicklung des DMP-RA konnte auf wesentliche Leitlinien der Deutschen Gesellschaft für Rheumatologie (DGRh) zurückgegriffen werden und DGRh-Experten waren als Sachverständige an der Erstellung des Programms beim G-BA beteiligt. «Das DMP ist ein strukturiertes Behandlungsprogramm, das Patienten durch eine frühe und moderne Behandlung eine Remission oder zumindest eine geringe Krankheitsaktivität sichern soll», erläutert Professor Specker, Direktor der Klinik für Rheumatologie \& Klinische Immunologie der Kliniken Essen-Mitte.
In der Regel bieten die gesetzlichen Krankenkassen in Zusammenarbeit mit Ärztinnen und Ärzten die DMP an. Patienten nehmen freiwillig daran teil. Ein DMP definiert Schnittstellen zwischen Haus- und Fachärzten und will so Patienten zu einer zügigen Diagnose und Behandlung verhelfen. Außerdem wird die Therapie der Patienten beim niedergelassenen Arzt, im Krankenhaus oder einer RehaEinrichtung koordiniert und stets an den individuellen Gesundheitszustand angepasst. Verschlechtert sich dieser, soll eine Überweisung an den Facharzt erfolgen. Die Patienten selbst sind aktiv involviert: «Indem Patientenschulungen in das DMP eingebunden sind, werden Menschen mit RA darin gestärkt, ihre chronische Erkrankung zu bewältigen und ihre Lebensqualität zu verbessern», lobt Professor Specker das Programm.

«Für die Patientenversorgung ist das DMP eine immense Chance», betont Professor Fleck, der als rheumatologischer Sachverständiger die Verhandlungen begleitet hat. «Wir hoffen, dass sich möglichst viele Rheu-

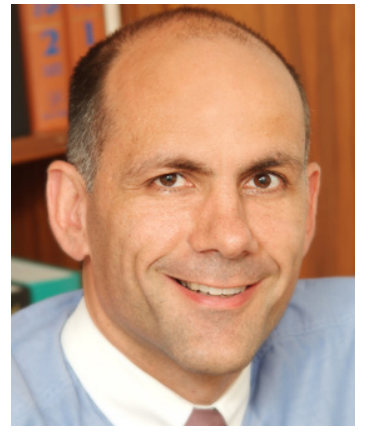

Prof. Christof Specker, Vizepräsident der DGRh, erhofft sich mehr Eigenverantwortung der Patienten.

matologinnen und Rheumatologen beteiligen, damit dieses DMP zu einem Erfolg wird!» Es könne noch ungefähr ein Jahr dauern, bis sich erste Patienten in das DMP einschreiben können. Auch wenn die Inhalte und Prozesse im DMP-RA nun definiert sind, müssen noch die praktischen Umsetzungen entwickelt und Verträge zwischen Krankenkassen und Praxen und Kliniken geschlossen werden.

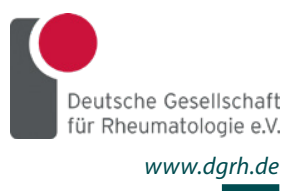

